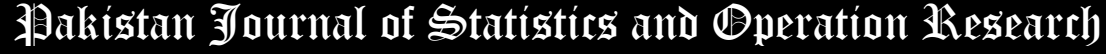

\section{The Modified Kumaraswamy Weibull Distribution: Properties and Applications in Reliability and Engineering Sciences}

\author{
Mohamed E. Mead ${ }^{1,2 *}$, Ahmed Z. Afify ${ }^{3}$, Nadeem Shafique Butt ${ }^{4}$ \\ * Corresponding Author \\ 1. University of Jeddah, College of Science and Arts at Khulis, Department of Mathematics, Jeddah, Saudi \\ Arabia, memead@uj.edu.sa \\ 2. Department of Statistics, Mathematics and Insurance, Zagazig University, Egypt, mead9990@gmail.com \\ 3. Department of Statistics, Mathematics and Insurance, Benha University, Egypt, ahmed.afify@fcom.bu.edu.eg \\ 4. Department of Family and Community Medicine, Rabigh Faculty of Medicine, King Abdul Aziz University, \\ Jeddah, Saudi Arabia, nshafique@kau.edu.sa
}

\begin{abstract}
We introduce the Kumaraswamy alpha power-G (KAP-G) family which extends the alpha power family (Mahdavi and Kundu, 2017) and some other families. We consider the Weibull as baseline for the KAP family and generate Kumaraswamy alpha power Weibull distribution which has right-skewed, left-skewed, symmetrical, and reversed$\mathbf{J}$ shaped densities, and decreasing, increasing, bathtub, upside-down bathtub, increasing-decreasing-increasing, $\mathbf{J}$ shaped and reversed-J shaped hazard rates. The proposed distribution can model non-monotone and monotone failure rates which are quite common in engineering and reliability studies. Some basic mathematical properties of the new model are derived. The maximum likelihood estimation method is used to evaluate the parameters and the observed information matrix is determined. The performance and flexibility of the proposed family is illustrated via two real data applications.
\end{abstract}

Key Words: Alpha power family; Kumaraswamy family; Maximum likelihood estimation; Weibull distribution.

Mathematical Subject Classification: 60E05, 62E15.

\section{Introduction}

Recently, Mahdavi and Kundu (2017) proposed the alpha power transformation (APT) family. For any baseline cumulative distribution function (CDF) $G(x)$, the APT family is specified by the CDF (for $x \in \mathcal{R}$ )

Its probability density function (PDF) has the form

$$
H_{A P T}(x)=\left\{\begin{array}{l}
\frac{\alpha^{G(x)}-1}{\alpha-1} \text { if } \alpha>0, \alpha \neq 1 \\
G(x) \text { if } \alpha=1 .
\end{array}\right.
$$

$$
h_{A P T}(x)=\left\{\begin{array}{l}
\frac{\ln \alpha}{\alpha-1} g(x) \alpha^{G(x)} \text { if } \alpha>0, \alpha \neq 1 \\
g(x) \text { if } \alpha=1 .
\end{array}\right.
$$

The APT family is considered by several authors to extend some distributions and to propose other extensions to it. For example, Mead et al. (2019) derived the general properties of the APT method and studied the alpha power exponentiated Weibull distribution. Nassar et al. (2019) introduced a new extension of the APT method called Marshall Olkin alpha power-G family.

In this paper, we study a new wider class called the Kumaraswamy alpha power-G (KAP-G) family, with three shape parameters to increase the flexibility to the generated class. The new KAP-G family is constructed based on the Kumaraswamy-G (K-G) family (Cordeiro and de Castro, 2011). 
Consider the CDF and PDF of a given random variable namely $G(x)$ and $g(x)$. Then, the CDFand PDF of the K-G family take the forms

and

$$
F(x)=1-\left[1-G(x)^{a}\right]^{b}, a, b>0
$$

$$
f(x)=\operatorname{abg}(x) G(x)^{a-1}\left[1-G(x)^{a}\right]^{b-1}, a, b>0 .
$$

The K-G family has been used to construct several extedned distributions and families. For example, the Kumaraswamy complementary Weibull geometric distribution (Afify et al., 2017), the Kumaraswamy transmuted-G family (Afify et al., 2016a).

We define a new KAP-G family by inserting the CDF of the APT as a baseline CDF in Equation (3). The new KAP$\mathrm{G}$ class is used to construct the new four-parameter KAP-Weibull (KAPW) distribution, which has several desirable properties.

The KAP-G family some useful motivations are: (i) Its special sub-models, for example the KAPW model, contains some lifetime sub-models such as the Weibull, exponentiated Weibull (Mudholkar and Srivastava, 1993) and Kumaraswamy Weibull (Cordeiro et al., 2010), among others; (ii) Its sub-models can be used in modeling all important hazard rate shapes such as bathtub, upside down bathtub, increasing, decreasing, and reversed-J hazard rates; and (iii) The KAPW model compares very well with other eight competing extensions of the Weibull distribution in two real data applications.

The rest of this paper is outlined as follows. We define the KAP-G distribution and its special cases are presented in Section 2. In Section 3, we study the new KAPW distribution. Some mathematical properties of the KAPW distribution are derived in Section 4. The maximum likelihood estimators of the model parameters are obtained, and some simulations are provided to assess the performance of these estimators in Section 5. In Section 6, we analyze two real data sets to illustrate the importance and flexibility of the KAPW model. Finally, some conclusions are given in Section 7.

\section{The KAP-G family}

The CDF of the KAP-G family follows by replacing $G(x)$ in Equation (1) by the CDF of the APT family (3), $H_{A P T}(x)$. Then, the CDF of KAP-G class has the form

$$
F(x)=\left\{\begin{array}{l}
1-\left\{1-\left[\frac{\alpha^{G(x)}-1}{\alpha-1}\right]^{a}\right\}^{b} \text { if } \alpha, a, b>0, \alpha \neq 1 \\
G(x) \text { if } \alpha=1
\end{array}\right.
$$

The KAP-G PDF can be expressed as

$$
f(x)=\left\{\begin{array}{l}
\frac{a b \ln (\alpha)}{\alpha-1} g(x) \alpha^{G(x)}\left[\frac{\alpha^{G(x)}-1}{\alpha-1}\right]^{a-1}\left\{1-\left[\frac{\alpha^{G(x)}-1}{\alpha-1}\right]^{a}\right\}^{b-1} \text { if } \alpha, a, b>0, \alpha \neq 1 \\
G(x) \text { if } \alpha=1 .
\end{array}\right.
$$

The quantile function $(\mathrm{QF})$ of $X, Q(p)=F^{-1}(p)$, can be obtained by inverting (5), as

$$
Q_{K A P}(p)=G^{-1}\left(\frac{\log \left\{1+(\alpha-1)\left[1-(1-p)^{1 / b}\right]^{1 / a}\right\}}{\log (\alpha)}\right), \alpha \neq 1 .
$$

A random sample of size $n$ the KAP-G CDF is obtained (for $\alpha \neq 1$ ), using the above equation, as $X_{i}=Q_{K A P}\left(U_{i}\right)$, where $U_{i} \sim \operatorname{Uniform}(0,1), i=1, \ldots, n$.

The new KAP-G class contains some special cases which are shown in Table 1.

Table 1: Sub-families of the KAP-G family

\begin{tabular}{|c|c|c|l|l|}
\hline \hline$\alpha$ & $a$ & $b$ & Reduced family & Authors \\
\hline \hline$\alpha$ & $a$ & 1 & Exponentiated alpha power-G (EAP-G) & New \\
\hline 1 & $a$ & $b$ & Kumaraswamy-G (K-G) & Cordeiro and de Castro (2011) \\
\hline$\alpha$ & 1 & 1 & Alpha power-G (AP-G) & Mahdavi and Kundu (2017) \\
\hline 1 & $a$ & 1 & Exponentiated-G (E-G) & Gupta et al. (1998) \\
\hline \hline
\end{tabular}

Using the following two power series

and

$$
\alpha^{q}=\sum_{k=0}^{\infty} \frac{(\ln \alpha)^{k}}{k !} q^{k}
$$




$$
(1-z)^{q}=\sum_{k=0}^{\infty}\left(\begin{array}{l}
q \\
k
\end{array}\right)(-1)^{k} z^{k}, q>0 .
$$

A useful mixture representation of the KAP-G density in (6) (for $\alpha>0, \alpha \neq 1$ ) follows as

Hence

$$
f(x)=\sum_{i=0}^{\infty} \frac{(-1)^{i}}{(\alpha-1)^{a(i+1)}}\left(\begin{array}{c}
b-1 \\
i
\end{array}\right)\left[\alpha^{G(x)}-1\right]^{a(i+1)-1} .
$$

Then, we can write

$$
\begin{aligned}
{\left[\alpha^{G(x)}-1\right]^{a(i+1)-1} } & =\alpha^{[a(i+1)-1] G(x)} \sum_{j=0}^{\infty}(-1)^{j}\left(\begin{array}{c}
a(i+1)-1 \\
j
\end{array}\right) \alpha^{-j G(x)} \\
& =\sum_{j=0}^{\infty}(-1)^{j}\left(\begin{array}{c}
a(i+1)-1 \\
j
\end{array}\right) \alpha^{[a(i+1)-j-1] G(x)}
\end{aligned}
$$

$$
f(x)=a b \ln (\alpha) g(x) \sum_{i, j=0}^{\infty} \frac{(-1)^{i+j}}{(\alpha-1)^{a(i+1)+1}}\left(\begin{array}{c}
b-1 \\
i
\end{array}\right)\left(\begin{array}{c}
a(i+1)-1 \\
j
\end{array}\right) \alpha^{[a(i+1)-j] G(x)} .
$$

Applying the power series

$$
\alpha^{q}=\sum_{k=0}^{\infty} \frac{(\ln \alpha)^{k}}{k !} q^{k}
$$

the PDF of the KAP-G class reduces to

Then, we have

$$
f(x)=a b \sum_{i, j, k=0}^{\infty} \frac{(-1)^{i+j}[a(i+1)-j]^{k}}{k !(\alpha-1)^{a(i+1)+1}[\ln (\alpha)]^{-k-1}} g(x) G(x)^{k}\left(\begin{array}{c}
b-1 \\
i
\end{array}\right)\left(\begin{array}{c}
a(i+1)-1 \\
j
\end{array}\right) .
$$

$$
f(x)=\sum_{k=0}^{\infty} \delta_{k} h_{k+1}(x)
$$

where $h_{k+1}(x)=(k+1) g(x) G(x)^{k}$ is the exponentiated-G density with power parameter and $(k+1)>0$ and

$$
\delta_{k}=a b \sum_{i, j=0}^{\infty} \delta_{k} \frac{(-1)^{i+j}[\ln (\alpha)]^{k+1}[a(i+1)-j]^{k}}{(k+1) !(\alpha-1)^{a(i+1)+1}}\left(\begin{array}{c}
b-1 \\
i
\end{array}\right)\left(\begin{array}{c}
a(i+1)-1 \\
j
\end{array}\right) .
$$

\section{The KAPW distribution}

The Weibull (W) distribution is considered one of the most popular life time distributions in the theory of reliability, and it has many applications in biological, medical, engineering, and meteorology, among others. Recently, many authors have proposed various generalized forms of the $\mathrm{W}$ distribution to increase its flexibility in modeling different types of lifetime data. For example, the exponentiated W distribution by Mudholkar and Srivastava (1993), the additive W distribution by Xie and Lai (1995), the modified W distribution by Xie et al. (2002), generalized modified W distribution by Jalmar et al. (2008), Kumaraswamy W distribution by Cordeiro et al. (2010), the exponential W distribution by Cordeiro et al. (2014), the alpha logarithmic transformed W distribution by Nassar et al. (2018) and the Lindley W distribution by Cordeiro et al. (2018).

Consider the two-parameter $\mathrm{W}$ distribution with a scale parameter $\lambda>0$ and a shape parameter $\beta>0$, then the CDF of the $\mathrm{W}$ random variable $Y$ has the form

and the corresponding PDF is

$$
F_{W}(y ; \lambda, \beta)=1-e^{-\lambda y^{\beta}}, y, \lambda, \beta>0
$$

$$
f_{W}(y ; \lambda, \beta)=\lambda \beta y^{\beta-1} e^{-\lambda y^{\beta}}, y, \lambda, \beta>0 .
$$

The $r$ th ordinary and incomplete moments of $Y$ are, respectively, expressed as

$$
\mu_{r, Y}^{\prime}=\lambda^{-\frac{r}{\beta}} \Gamma\left(\frac{r}{\beta}+1\right), \quad \varphi_{r, Y}(t)=\lambda^{-\frac{r}{\beta}} \gamma\left(\frac{r}{\beta}+1, \lambda t^{\beta}\right),
$$

where $\gamma(a, b)=\int_{0}^{b} x^{a-1} e^{-x} d x$ refers to lower incomplete gamma function. Inserting (7) in Equation (5), the CDF of the KAPW distribution is 


$$
F(x, \varphi)= \begin{cases}1-\left[1-\left(\frac{\alpha^{1-e^{-\lambda x^{\beta}}}-1}{\alpha-1}\right)^{a}\right]^{b} & \text { if } \alpha \neq 1 \\ 1-\left[1-\left(1-e^{-\lambda x^{\beta}}\right)^{a}\right]^{b} & \text { if } \alpha=1 .\end{cases}
$$

The PDF corresponding to (9) is

$$
f(x, \varphi)= \begin{cases}\frac{a b \lambda \beta(\ln \alpha)}{\alpha-1} x^{\beta-1} e^{-\lambda x^{\beta}} \alpha^{1-e^{-\lambda x \beta}}\left(\frac{\alpha^{1-e^{-\lambda x \beta}}-1}{\alpha-1}\right)^{a-1}\left[1-\left(\frac{\alpha^{1-e^{-\lambda x^{\beta}}}-1}{\alpha-1}\right)^{a}\right]^{b-1} & \text { if } \alpha \neq 1 \\ a b \lambda \beta e^{-\lambda x^{\beta}}\left(1-e^{-\lambda x^{\beta}}\right)^{a-1}\left[1-\left(1-e^{-\lambda x^{\beta}}\right)^{a}\right]^{b-1} & \text { if } \alpha=1\end{cases}
$$

The hazard rate function (HRF) of $X$ are, respectively, given by

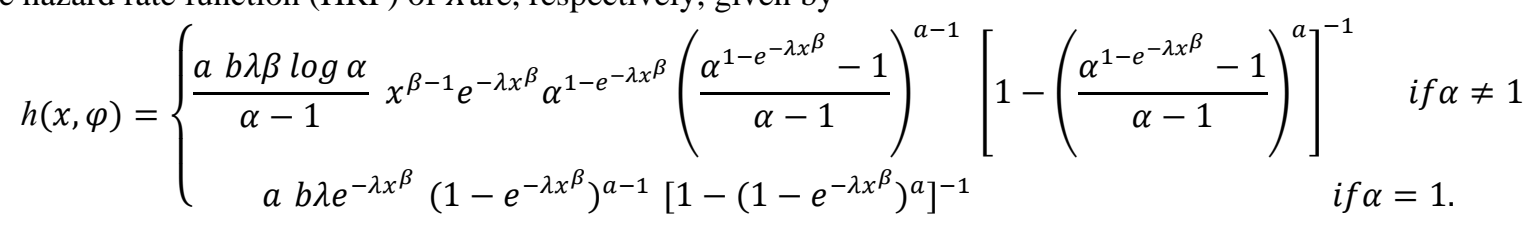

Table 2 lists seventeen important special models of the new distribution.

Figures 1 and 2 display some shapes of the KAPW density function for some selected parameter values. Some HRF shapes of the KAPW distribution for some parameter values are plotted in Figure 3.

Table 2: Sub-models of the KAPW distribution

\begin{tabular}{|c|c|c|c|c|l|l|}
\hline \hline$\alpha$ & $a$ & $b$ & $\lambda$ & $\beta$ & Reduced model & Authors \\
\hline \hline$\alpha$ & $a$ & $b$ & $\lambda$ & 2 & KAP-Rayleigh & New \\
\hline$\alpha$ & $a$ & $b$ & $\lambda$ & 1 & KAP-exponential & New \\
\hline 1 & $a$ & $b$ & $\lambda$ & $\beta$ & K-Weibull & Cordeiro et al. (2010) \\
\hline 1 & $a$ & $b$ & $\lambda$ & 2 & K-Rayleigh & - \\
\hline 1 & $a$ & $b$ & $\lambda$ & 1 & K-exponential & - \\
\hline 1 & $a$ & 1 & $\lambda$ & $\beta$ & E-Weibull & Mudholkar and Srivastava (1993) \\
\hline 1 & $a$ & 1 & $\lambda$ & 2 & E-Rayleigh & \\
\hline 1 & $a$ & 1 & $\lambda$ & 1 & E-exponential & \\
\hline$\alpha$ & $a$ & 1 & $\lambda$ & $\beta$ & EAP-Weibull & \\
\hline$\alpha$ & $a$ & 1 & $\lambda$ & 2 & EAP-Rayleigh & \\
\hline$\alpha$ & $a$ & 1 & $\lambda$ & 1 & EAP-exponential & \\
\hline$\alpha$ & 1 & 1 & $\lambda$ & $\beta$ & AP-Weibull & \\
\hline$\alpha$ & 1 & 1 & $\lambda$ & 2 & AP-Rayleigh & \\
\hline$\alpha$ & 1 & 1 & $\lambda$ & 1 & AP-exponential & \\
\hline 1 & 1 & 1 & $\lambda$ & $\beta$ & Weibull & \\
\hline 1 & 1 & 1 & $\lambda$ & 2 & Rayleigh & \\
\hline 1 & 1 & 1 & $\lambda$ & 1 & Exponential & \\
\hline \hline
\end{tabular}




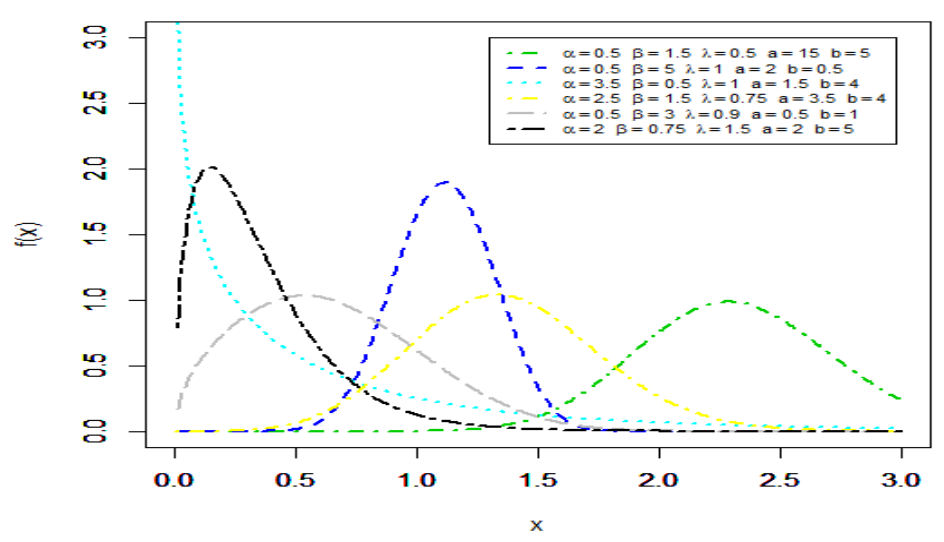

Figure 1: Shapes of the KAPW density for various parameter values

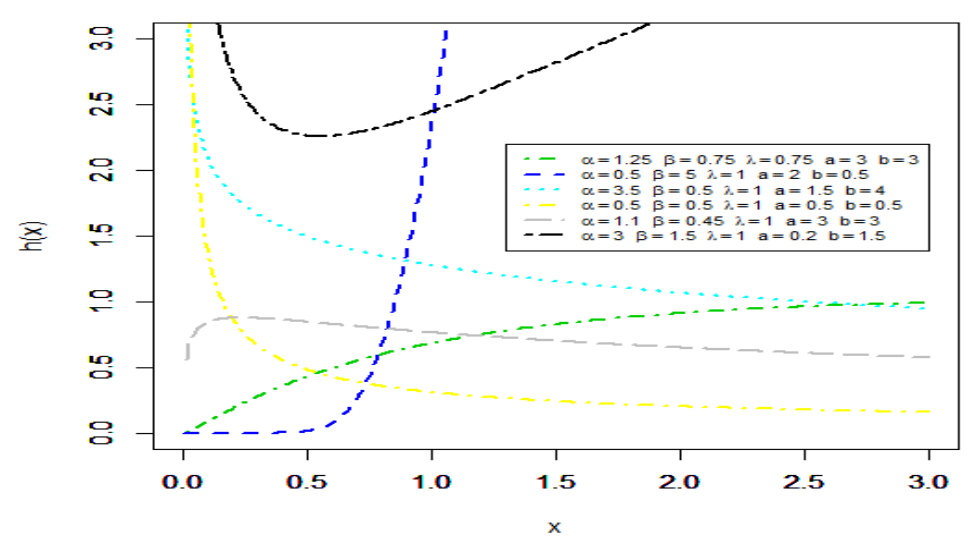

Figure 2: Shapes of the KAPW HRF for various parameters values

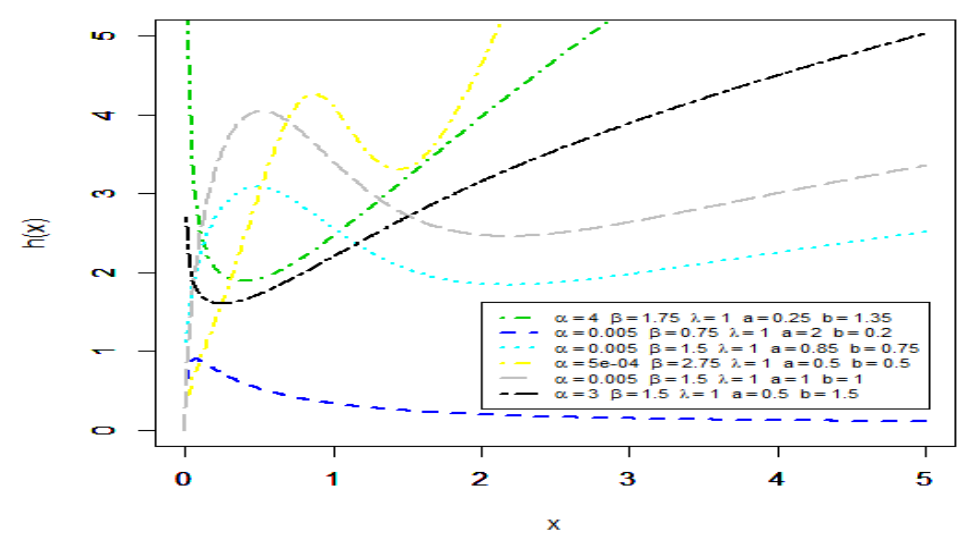

Figure 3: Shapes of the KAPW HRF for various parameters values

\section{Properties of KAPW distribution}

4.1 Linear representation

The KAPW density is expressed as a linear mixture of $\mathrm{W}$ densities 


$$
\begin{aligned}
f(x)=a b & \sum_{i, j, k=0}^{\infty} \frac{(-1)^{i+j}[a(i+1)-j]^{k}}{k !(\alpha-1)^{a(i+1)+1}[\ln (\alpha)]^{-k-1}}\left(\lambda \beta x^{\beta-1} e^{-\lambda x^{\beta}}\right) \\
& \times\left(1-e^{-\lambda x^{\beta}}\right)^{k}\left(\begin{array}{c}
b-1 \\
i
\end{array}\right)\left(\begin{array}{c}
a(i+1)-1 \\
j
\end{array}\right) .
\end{aligned}
$$

Applying the binomial expansion to $\left(1-e^{-\lambda x^{\beta}}\right)^{k}$, we can write

$$
f(x)=a b \sum_{i, j, k=0}^{\infty} \sum_{m=0}^{k} \frac{(-1)^{i+j+m}[a(i+1)-j]^{k}}{k !(\alpha-1)^{a(i+1)+1}[\ln (\alpha)]^{-k-1}} \lambda \beta x^{\beta-1} e^{-(m+1) \lambda x^{\beta}}\left(\begin{array}{c}
b-1 \\
i
\end{array}\right)\left(\begin{array}{c}
a(i+1)-1 \\
j
\end{array}\right)\left(\begin{array}{l}
k \\
m
\end{array}\right) .
$$

Then, we have

$$
f(x)=\sum_{m=0}^{\infty} d_{m} g_{m+1}(x ; \beta,(m+1) \lambda),
$$

where $g_{m+1}(x ; \beta,(m+1) \lambda)$ refers to the W PDF with parameters $\beta$ and $(m+1) \lambda$, and $d_{m}$ is the constant term

$$
d_{m}=\sum_{i, j,=0}^{\infty} \sum_{k=m}^{\infty} \frac{(-1)^{i+j+m} a b[a(i+1)-j]^{k}}{k !(m+1)(\alpha-1)^{a(i+1)+1[\ln (\alpha)]^{-k-1}}}\left(\begin{array}{c}
b-1 \\
i
\end{array}\right)\left(\begin{array}{c}
a(i+1)-1 \\
j
\end{array}\right)\left(\begin{array}{l}
k \\
m
\end{array}\right) .
$$

Equation (11) shows that the KAPW PDF is written as a linear mixture of W densities. Then, several of its properties can be obtained from the properties of the $\mathrm{W}$ distribution.

4.2 Quantile Function

The QF of the KAPW distribution follows by inverting Equation (9) as

$$
Q(u)=\left[\frac{-1}{\lambda} \log \left(1-\frac{\log \left\{1+(\alpha-1)\left[1-(1-u)^{1 / b}\right]^{1 / a}\right\}}{\log (\alpha)}\right)\right]^{1 / \beta},
$$

Where $U$ follows uniform $(0,1)$ distribution.

\subsection{Moments}

The $r$ th moment of $X$ can be obtained from Equation (11) as

$$
E\left(X^{r}\right)=\sum_{m=0}^{\infty} d_{m}[(m+1) \lambda]^{-\frac{r}{\beta}} \Gamma\left(\frac{r}{\beta}+1\right)
$$

The $r$ th incomplete moment of the KAPW model, $\varphi_{r}(t)$, is given by $\varphi_{r}(t)=\int_{0}^{t} x^{r} f(x) d x$. Using Equation (11), $\varphi_{r}(t)$ reduces to

and then, we obtain

$$
\varphi_{r}(t)=\sum_{m=0}^{\infty} d_{m} \int_{0}^{t} x^{r} g_{m+1}(x) d x
$$

$$
\varphi_{r}(t)=\sum_{m=0}^{\infty} d_{m}[(m+1) \lambda]^{-\frac{r}{\beta}} \gamma\left(\frac{r}{\beta}+1,[(m+1) \lambda] t^{\beta}\right) .
$$

The first incomplete moment of $X, \varphi_{1}(t)$, is obtaind by replacing $r=1$ in the above equation.

\subsection{Moment generating function}

We now provide the moment generating function (MGF) of the $\mathrm{W}$ model as derived by Nadarajah et al. (2013). The MGF of Y can be expressed as

$$
M_{Y}(t ; \beta, \lambda)=\beta \lambda \int_{0}^{\infty} e^{t x} x^{\beta-1} e^{-\lambda x^{\beta}} d x
$$

By expanding $e^{t x}$ andcalculating the integral, we have

$$
M_{Y}(t ; \beta, \lambda)=\sum_{k=0}^{\infty} \frac{\left(t / \lambda^{1 / \beta}\right)^{k}}{k !} \Gamma\left(\frac{k}{\beta}+1\right) .
$$

Using the Wright generalized hypergeometric function defined by

Hence, we can write the MGF of $Y$ as

$$
{ }_{p} \Psi_{q}\left[\begin{array}{l}
\left(\lambda_{1}, A_{1}\right), \ldots,\left(\lambda_{p}, A_{p}\right) \\
\left(\beta_{1}, B_{1}\right), \ldots,\left(\beta_{p}, B_{p}\right)
\end{array} ; x\right]=\sum_{m=0}^{\infty} \frac{\prod_{j=1}^{p} \Gamma\left(\lambda_{j}+A_{j} m\right)}{\prod_{j=1}^{q} \Gamma\left(\beta_{j}+B_{j} m\right)} \frac{x^{m}}{m !} .
$$




$$
M_{Y}(t ; \beta, \lambda)={ }_{1} \Psi_{0}\left[\begin{array}{c}
\left(1,-\beta^{-1}\right) \\
-
\end{array} t / \lambda^{1 / \beta}\right]
$$

Combining the above expression and Equation (11), the MGF of $X$ can be expressed as

\subsection{Residual and reversed residual Lifes}

$$
M_{X}(t ; \beta, \lambda)=\sum_{m=0}^{\infty} d_{m}{ }_{1} \Psi_{0}\left[\begin{array}{c}
\left(1,-\beta^{-1}\right) \\
-
\end{array} t /[(m+1) \lambda]^{1 / \beta}\right]
$$

For $n=1,2, \ldots$ and $t>0$, the $n$th moment of residual life of $X$ has the form

Using Equation (11), we can write

$$
m_{n}(t)=\frac{1}{1-F(t)} \int_{t}^{\infty}(x-t)^{n} d F(x)
$$

$$
m_{n}(t)=\frac{1}{\bar{F}(t)} \sum_{m=0}^{\infty} \sum_{i=0}^{n} \frac{(n+1)_{i} t^{n-i}}{(-1)^{n-i} i !} d_{m}[(m+1) \lambda]^{-\frac{r}{\beta}} \gamma\left(\frac{r}{\beta}+1,[(m+1) \lambda] z^{\beta}\right),
$$

where $\rho_{i}=\Gamma(\rho+1) / \Gamma(\rho-i+1)$ refers to falling factorial.

The mean residual life refers to the expected additional life length for a unit which is alive at age $x$, and it follows for $X$ from the last equation with $n=1$.

For $n=1,2, \ldots$ and $t>0$, the $n$th moment of reversed residual life of the KAPW model takes the form

Then, we can write

$$
M_{n}(t)=\frac{1}{F(t)} \int_{0}^{t}(t-x)^{n} d F(x)
$$

$$
M_{n}(t)=\frac{1}{F(t)} \sum_{i=0}^{n} \sum_{m=0}^{\infty} \frac{(n+1)_{i} t^{n-i}}{(-1)^{i} i !} d_{m}[(m+1) \lambda]^{-\frac{r}{\beta}} \gamma\left(\frac{r}{\beta}+1,[(m+1) \lambda] z^{\beta}\right)
$$

The mean inactivity time refers to the waiting time elapsed since the failure of an item on condition that this failure had occurred in $(0, x)$, and it follows for $X$ from the above equation with $n=1$.

\subsection{Order statistics}

Let $X_{1}, X_{2}, \ldots, X_{n}$ be a random sample of size $n$, and let $X_{i: n}$ denote the $i$ th order statistic. The PDF of $X_{i: n}, f_{i: n}(x)$, is given by

$$
f_{i: n}(x)=\frac{n ! f(x)}{(i-1) !(n-i) !}[1-F(x)]^{n-i}[F(x)]^{i-1} .
$$

Substituting (9) and (10) in (12), the $i$ th order statistic of the KAPW distribution reduces to

$$
\begin{gathered}
f_{i: n}(x)=\frac{a b \lambda \beta \ln \alpha}{\beta(i, n-i+1)} x^{\beta-1} e^{-\lambda x^{\beta}} \alpha^{1-e^{-\lambda x^{\beta}}}\left(\frac{\alpha^{1-e^{-\lambda x^{\beta}}}-1}{\alpha-1}\right)^{a-1} \\
\times\left[1-\left(\frac{\alpha^{1-e^{-\lambda x^{\beta}}}-1}{\alpha-1}\right)^{a}\right]^{b(n-i+1)-1}\left[1-\left(1-\left(\frac{\alpha^{1-e^{-\lambda x^{\beta}}-1}}{\alpha-1}\right)^{a}\right)^{b}\right]^{i-1}
\end{gathered}
$$

Applying the binomial series, then $f_{i: n}(x)$ can be in the form

$$
\begin{aligned}
& f_{i: n}(x)=\sum_{k=0}^{i-1} \sum_{j, m, s=0}^{\infty} \sum_{l=0}^{s} \frac{(-1)^{k+j+m} a b(\ln \alpha)^{s+1}[a(j+1)-m]^{s}}{\beta(i, n-i+1) s !(\alpha-1)^{a(j+1)}}\left(\begin{array}{c}
i-1 \\
k
\end{array}\right)\left(\begin{array}{c}
b(n+k-i+1)-1 \\
j
\end{array}\right) \\
& \times\left(\begin{array}{c}
a(j+1)-1 \\
m
\end{array}\right)\left(\begin{array}{l}
s \\
l
\end{array}\right) \lambda \beta x^{\beta-1} e^{-(l+1) \lambda x^{\beta}} .
\end{aligned}
$$

Or simply in the form

$$
f_{i: n}(x)=\sum_{l=0}^{\infty} d_{l} g_{l+1}(x ; \beta,(l+1) \lambda),
$$

where $g_{l+1}(x ; \beta,(l+1) \lambda)$ as before, is $\mathrm{W}$ density with parameters $\beta$ and $(l+1) \lambda$, and

$$
=\sum_{k=0}^{d_{l}} \sum_{j, m=0}^{\infty} \sum_{s=l}^{\infty} \frac{a b(\ln \alpha)^{s+1}[a(j+1)-m]^{s}}{\beta(i, n-i+1) s !(\alpha-1)^{a(j+1)}}\left(\begin{array}{c}
i-1 \\
k
\end{array}\right)\left(\begin{array}{c}
b(n+k-i+1)-1 \\
j
\end{array}\right)\left(\begin{array}{c}
a(j+1)-1 \\
m
\end{array}\right)\left(\begin{array}{c}
s \\
l
\end{array}\right) \frac{(-1)^{k+j+m}}{(l+1)}
$$


The $q$ th moments of $X_{i: n}$ has the form

$$
E\left(X_{i: n}^{q}\right)=\sum_{l=0}^{\infty} d_{l}[(l+1) \lambda]^{-\frac{r}{\beta}} \Gamma\left(\frac{r}{\beta}+1\right) .
$$

\section{Estimation and simulations}

Let $x_{1}, x_{2}, \ldots, x_{n}$ be a random sample from KAPW distribution then the logarithm of the likelihood function $(\ell)$, becomes

$$
\begin{gathered}
\ell=n[\ln a+\ln b+\ln \lambda+\ln \beta]+n \ln \left(\frac{\ln \alpha}{\alpha-1}\right)+(\beta-1) \sum_{i=1}^{n} \ln x_{i}-\lambda \sum_{i=1}^{n} x_{i}^{\beta}+\ln (\alpha) \sum_{i=1}^{n} d_{i} \quad+(a- \\
\text { 1) } \sum_{i=1}^{n \sum\left(\xi_{i}\right) \sum_{i=1}^{n \sum\left[1-\xi_{i}{ }^{a}\right]} \ln l n} \ln
\end{gathered}
$$

where $d_{i}=1-e^{-\lambda x_{i}{ }^{\beta}}$ and $\xi_{i}=\left(\alpha^{d_{i}}-1\right) /(\alpha-1)$.

To obtain the MLEs of $a, b, \alpha, \lambda$ and $\beta$, the first derivatives of $\ell$ are obtained with respect to $a, b, \alpha, \lambda$ and $\beta$. These derivatives are

$$
\begin{gathered}
\frac{\partial \ell}{\partial a}=\frac{n}{a}+\sum_{i=1}^{n \sum\left(\xi_{i}\right) \sum_{i=1}^{n \sum\left(\xi_{i}\right)} \xi_{i}^{a}\left(1-\xi_{i}{ }^{a}\right)^{-1} l n} \\
\frac{\partial \ell}{\partial b}=\frac{n}{b}+\sum_{i=1}^{n \sum\left(1-\xi_{i}{ }^{a}\right)} \ln \\
\frac{\partial \ell}{\partial \alpha}=\frac{n[\alpha-1-\alpha \ln \alpha]}{\alpha(\alpha-1) \ln \alpha}+\frac{1}{\alpha} \sum_{i=1}^{n} d_{i}+(a-1) \sum_{i=1}^{n}\left[\frac{(\alpha-1) d_{i} \alpha^{-e^{-\lambda x^{\beta}}}-\left(\alpha^{d_{i}}-1\right)}{(\alpha-1)\left(\alpha^{d_{i}}-1\right)}\right]-a(b \\
-1) \sum_{i=1}^{n} \xi_{i}{ }^{a}\left[\frac{(\alpha-1) d_{i} \alpha^{-e^{-\lambda x^{\beta}}}-\left(\alpha^{d_{i}}-1\right)}{(\alpha-1)\left(\alpha^{d_{i}}-1\right)}\right]\left(1-\xi_{i}{ }^{a}\right), \\
\frac{\partial \ell}{\partial \lambda}=\frac{n}{\lambda}-\sum_{i=1}^{n} x_{i}^{\beta}+\ln \alpha \sum_{i=1}^{n} x_{i}^{\beta} e^{-\lambda x^{\beta}}+(a-1) \sum_{i=1}^{n}\left(\frac{\alpha^{d_{i}} x_{i}^{\beta} e^{-\lambda x^{\beta}} \ln \alpha}{\alpha^{d_{i}-1}}\right) \\
-a(b-1) \sum_{i=1}^{n} \xi_{i}^{a}\left(\frac{\alpha^{d_{i}} x_{i}^{\beta} e^{-\lambda x^{\beta}} \ln \alpha}{\alpha^{d_{i}}-1}\right)\left(1-\xi_{i}{ }^{a}\right)^{-1}
\end{gathered}
$$

and

$$
\begin{aligned}
& \frac{\partial \ell}{\partial \beta}=\frac{n}{\beta}+\sum_{\sum_{i} \sum_{i=1}^{n} x_{i}^{\beta} \ln x_{i} \ln \alpha \sum_{i=1}^{n} \lambda x_{i}^{\beta^{-\lambda x^{\beta}}} \ln x_{i} \sum_{i=1}^{n}\left(\frac{\alpha^{d_{i} \lambda x_{i}^{\beta} e^{-\lambda x^{\beta}} \ln \alpha \ln x_{i}}}{\alpha^{d_{i-1}}}\right)} \sum_{i=1}^{n} \ln \sum_{i=1}^{n} \xi_{i}^{a}\left(\frac{\alpha^{d_{i}} \lambda x_{i}^{\beta} e^{-\lambda x^{\beta}} \ln \alpha \ln x_{i}}{\alpha^{d_{i}}-1}\right)\left(1-\xi_{i}^{a}\right)^{-1} .
\end{aligned}
$$

The MLEs of the parameters $a, b, \alpha, \lambda$ and $\beta$ can follow by solving the above system of equations. No explicit form for these estimates, we use a numerical technique like Newton-Raphson method is used to solve the above non-linear equations.

Now we investigate the performance of the of maximum likelihood estimators for the KAPW parameters. We generate 5,000 samples of the KAPW distribution using its QF in Section 4.2, for some sample sizes, $n=(20,50,100,200,400)$, and for some parameters values, where $\alpha=(0.75,2.75), \beta=(0.67,2.00,2.5), \lambda=(.5,0.67,2.50), a=(0.25,0.75,1.5)$ and $b=(0.30,0.5,1.50)$.

For each each sample and parameters combination, we use the $\mathrm{R}$ software to obtain the avagrage values of the estimates (AEs), mean squared errors (MSEs), biases and mean relative estimates (MREs). Tables 3, 4 and 5 show the AEs, MSEs, bias and MREs of the MLEs for the KAPW parameters. It is clear that the values of MSEs, biases and MREs decrease as 
$n$ increases for all cases, which illustrates the estimators are quite stable and the estimates are very close to the true values of the parameters.

\section{Applications}

In this section, the importance and flexibility KAPW distribution are studied via two real data applications. The first set of data refers to the actual taxes data that represent monthly actual taxes revenue in Egypt from January 2006 to November 2010 (taxes revenue in 1000 million Egyptian pounds).

The second set of data represents strengths of $1.5 \mathrm{~cm}$ glass fibers of 63 observations which originally obtained by workers at the UK National Physical Laboratory. These data were reported in Smith and Naylor (1987) and analyzed by Afify et al. (2016b) and Alizadeh et al. (2020).

For both data sets, we compare the fits of the KAPW distribution with some competitive models called, generalized Burr X W (GBXW) by Aldahlan et al. (2018), exponentiated W (EW) by Mudholkar and Srivastava (1993), odd loglogistic exponentiated W (OLLEW) by Afify et al. (2018), alpha power W (APW) by Nassar et al. (2017), transmuted complementary W geometric (TCWG) by Afify et al. (2014), alpha logarithmic transformed W by Nassar et al. (2018), W-W (WW) by Abouelmagd et al. (2017) and W distributions.

Table 3: The AEs, MSEs, biases and MREs of the KAPW parameters for different values of the parameter and n

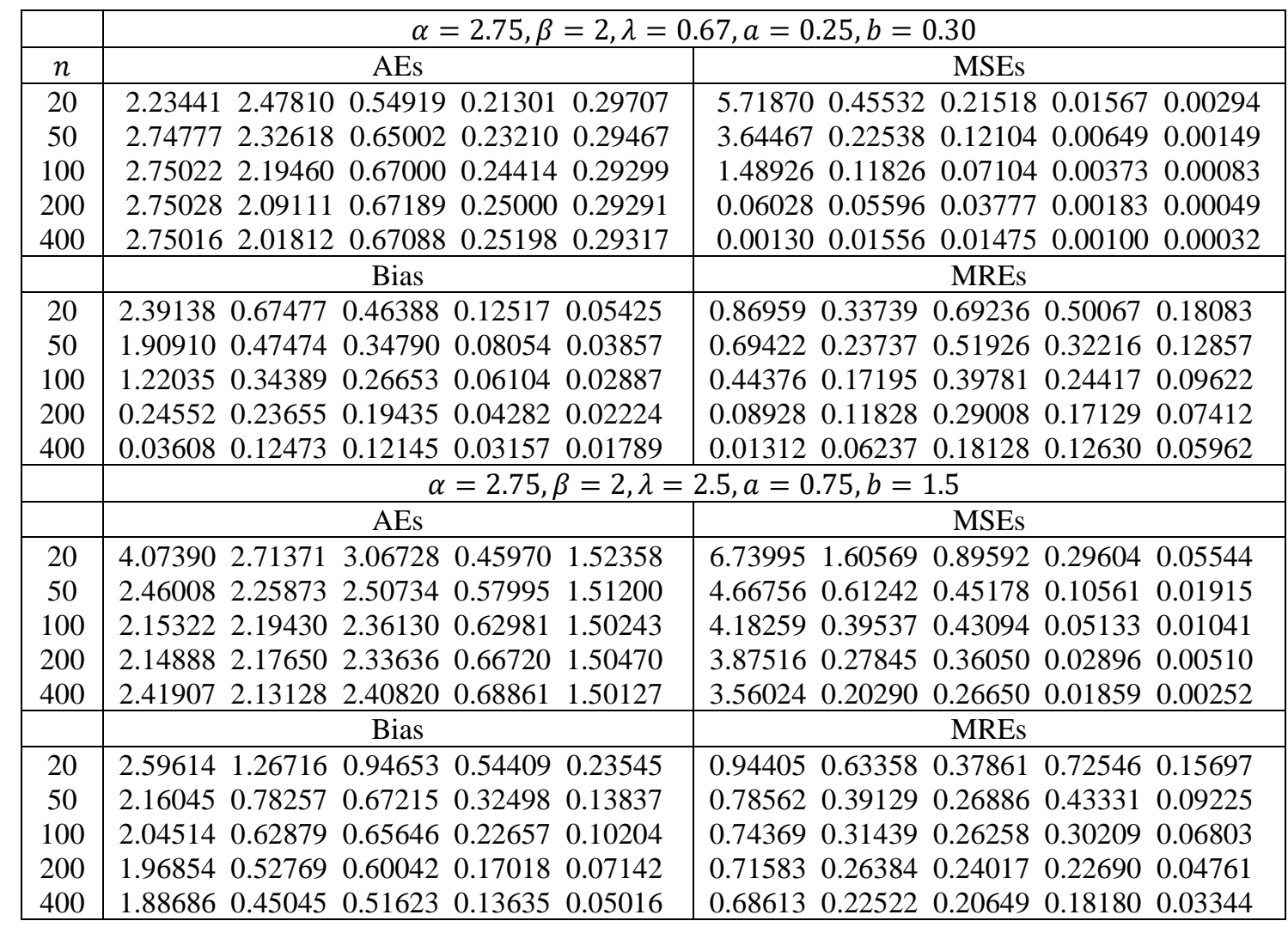


Table 4: The AEs, MSEs, biases and MREs of the KAPW parameters for different values of the parameter and $n$

\begin{tabular}{|c|c|c|c|c|c|c|c|}
\hline & \multicolumn{7}{|c|}{$\alpha=0.75, \beta=2.5, \lambda=0.67, a=0.25, b=0.5$} \\
\hline$n$ & \multicolumn{3}{|c|}{ AEs } & \multicolumn{4}{|c|}{ MSEs } \\
\hline 20 & 0.696672 .90606 & 0.50646 & 0.206960 .50971 & 0.371340 .64446 & 0.14248 & 0.01095 & 0.00643 \\
\hline 50 & 0.704482 .67970 & 0.58261 & 0.228430 .49982 & 0.206210 .22990 & 0.07847 & 0.00413 & 0.00274 \\
\hline 100 & 0.729342 .56191 & 0.63977 & 0.237870 .49862 & 0.130560 .11069 & 0.04260 & 0.00198 & 0.00137 \\
\hline 200 & 0.739502 .50304 & 0.66615 & $0.24514 \quad 0.49733$ & 0.063650 .04431 & 0.01913 & 0.00096 & 0.00072 \\
\hline 400 & 0.742902 .49686 & 0.67027 & $0.24927 \quad 0.49810$ & $0.00990 \quad 0.00764$ & 0.00288 & 0.00036 & 0.00035 \\
\hline & \multicolumn{3}{|c|}{ Bias } & \multicolumn{4}{|c|}{ MREs } \\
\hline 20 & 0.609380 .80278 & 0.37746 & 0.104640 .08017 & $0.81250 \quad 0.32111$ & 0.56338 & 0.41857 & 0.16035 \\
\hline 50 & 0.454100 .47948 & 0.28012 & 0.064300 .05231 & 0.605470 .19179 & 0.41809 & 0.25720 & 0.10461 \\
\hline 100 & $0.36134 \quad 0.33270$ & 0.20640 & 0.044480 .03695 & 0.481780 .13308 & 0.30806 & 0.17792 & 0.07390 \\
\hline 200 & 0.252280 .21049 & 0.13832 & 0.031010 .02684 & 0.336380 .08420 & 0.20645 & 0.12402 & 0.05369 \\
\hline \multirow[t]{3}{*}{400} & $0.09948 \quad 0.08743$ & 0.05364 & 0.018920 .01877 & 0.132640 .03497 & 0.08005 & 0.07566 & 0.03754 \\
\hline & \multicolumn{7}{|c|}{$\alpha=0.75, \beta=0.35, \lambda=0.5, a=1.65, b=0.5$} \\
\hline & \multicolumn{3}{|c|}{ AEs } & \multicolumn{4}{|c|}{ MSEs } \\
\hline 20 & 0.283720 .41121 & 0.32293 & $1.43652 \quad 0.44848$ & $0.45634 \quad 0.01502$ & 0.14421 & 1.34102 & 0498 \\
\hline 50 & 0.343320 .39950 & 0.34758 & 1.342310 .44909 & 0.403700 .00767 & 0.09511 & 0.64379 & 0.00197 \\
\hline 100 & 0.438850 .39409 & 0.38444 & 1.413910 .44913 & 0.370300 .00590 & 0.07276 & 0.37001 & 0.00103 \\
\hline 200 & 0.634810 .39751 & 0.44257 & 1.512450 .44598 & $0.30684 \quad 0.00527$ & 0.04902 & 0.18013 & 0.00065 \\
\hline 400 & $0.75501 \quad 0.40141$ & 0.47861 & 1.674710 .44313 & 0.165820 .00479 & 0.02023 & 0.05996 & 0.00045 \\
\hline & \multicolumn{3}{|c|}{ Bias } & \multicolumn{4}{|c|}{ MREs } \\
\hline 20 & 0.675530 .12254 & 0.37975 & $1.15802 \quad 0.07059$ & $0.90070 \quad 0.35012$ & 0.77501 & 0.66173 & 0.15686 \\
\hline 50 & 0.635370 .08760 & 0.30841 & 0.802370 .04439 & 0.847160 .25028 & 0.62940 & 0.45849 & 0.09864 \\
\hline 100 & 0.608530 .07684 & 0.26974 & $0.60828 \quad 0.03210$ & $0.81137 \quad 0.21954$ & 0.55050 & 0.34759 & 0.07133 \\
\hline 200 & 0.553940 .07257 & 0.22141 & $0.42442 \quad 0.02540$ & 0.738580 .20735 & 0.45186 & 0.24252 & 0.05645 \\
\hline 400 & $0.40721 \quad 0.06922$ & 0.14224 & 0.244860 .02117 & $0.54295 \quad 0.19777$ & 0.29029 & 0.13992 & 0.04704 \\
\hline
\end{tabular}

Table 5: The AEs, MSEs, biases and MREs of the KAPW parameters for different values of the parameter and $n$

\begin{tabular}{|c|c|c|c|c|c|c|c|}
\hline & \multicolumn{7}{|c|}{$\alpha=0.75, \beta=0.67, \lambda=2.5, a=1.5, b=0.30$} \\
\hline$n$ & \multicolumn{3}{|c|}{ AEs } & \multicolumn{4}{|c|}{ MSEs } \\
\hline 20 & $0.82813 \quad 1.60719$ & 2.48213 & 1.633780 .00000 & $0.02958 \quad 0.87833$ & 0.00209 & 0.06527 & 0.0900 \\
\hline 50 & 0.830631 .54315 & 2.48371 & 1.615880 .00000 & 0.019900 .76240 & 0.00128 & 0.04502 & 0.0900 \\
\hline 100 & $0.81852 \quad 1.45496$ & 2.48487 & 1.583730 .00000 & $0.01740 \quad 0.61616$ & 0.00219 & 0.03662 & 0.0900 \\
\hline 200 & $0.79430 \quad 0.98983$ & 2.48992 & 1.541650 .25230 & 0.015840 .10229 & 0.00293 & 0.02783 & 0.0046 \\
\hline \multirow[t]{2}{*}{400} & 1780.76367 & 2.49898 & 1.502850 .28154 & $0.00988 \quad 0.01257$ & 0.00284 & 0.01591 & 0.0006 \\
\hline & \multicolumn{3}{|c|}{ Bias } & \multicolumn{4}{|c|}{ MREs } \\
\hline 20 & $0.17198 \quad 0.93719$ & 0.04570 & 0.255490 .30000 & 0.229311 .39880 & 0.01828 & 32 & 000 \\
\hline 50 & 1080.87 & 0.03578 & $0.21217 \quad 0.30000$ & 0.188101 .30322 & 0.01431 & 145 & 000 \\
\hline 100 & 131920.78496 & 0.04679 & 0.191350 .30000 & 0.175901 .17158 & 0.01872 & 0.12757 & 1.00000 \\
\hline 200 & 5860.31983 & 0.05411 & 0.166820 .06782 & 0.167820 .47736 & 0.02164 & 0.11121 & 0.22608 \\
\hline 400 & $0.09938 \quad 0.11212$ & 0.05329 & 0.126150 .02454 & 0.132510 .16734 & 0.02132 & 0.08410 & 0.08180 \\
\hline & \multicolumn{7}{|c|}{$\alpha=0.75, \beta=2, \lambda=0.5, a=0.75, b=1.5$} \\
\hline & \multicolumn{3}{|c|}{ AEs } & \multicolumn{4}{|c|}{ MSEs } \\
\hline 20 & $0.75302 \quad 2.40342$ & 0.35233 & $0.58962 \quad 1.52773$ & 0.425591 .36032 & 0.186960 & 9570 & .05411 \\
\hline 50 & 0.708632 .17464 & 0.38382 & $0.67427 \quad 1.50176$ & 0.184440 .41931 & $0.10055 \mathrm{C}$ & 630 & .02095 \\
\hline 100 & 0.701802 .09288 & 0.42113 & $0.70317 \quad 1.50325$ & 0.131110 .22170 & $0.06782 \mathrm{C}$ & 0.05 & .01026 \\
\hline 200 & 0.704012 .09670 & 0.42683 & $0.71209 \quad 1.50284$ & 0.113520 .14098 & $0.04963 \mathrm{C}$ & 0. & 524 \\
\hline 400 & 0.697712 .05928 & 0.45901 & $0.72852 \quad 1.50138$ & 0.103950 .10081 & $0.03952 \mathrm{C}$ & 8920 & .00260 \\
\hline & \multicolumn{3}{|c|}{ Bias } & \multicolumn{4}{|c|}{ MREs } \\
\hline 20 & $0.65237 \quad 1.166$ & 0.43239 & 0.556390 .23 & 0.869830 .58316 & 0.86477 & 85 & \\
\hline 50 & $2947 \quad 0.64754$ & 0.31710 & 0.331110 .14 & 0.572630 .32377 & 0.63420 & 0.44148 & 0.09649 \\
\hline 100 & 0.362090 .47085 & 0.26042 & 0.224580 .10130 & 0.482780 .23542 & 0.52084 & 0.29944 & 0.06754 \\
\hline 200 & 0.336930 .37547 & 0.22279 & $0.16643 \quad 0.072$ & 0.449240 .18774 & 0.44557 & 0.22190 & 0.04826 \\
\hline 400 & 0.352060 .31750 & 0.19880 & $\begin{array}{lll}0.13754 & 0.05097\end{array}$ & $\begin{array}{lll}0.46941 & 0.15875\end{array}$ & 0.39761 & 0.18338 & 0.03398 \\
\hline
\end{tabular}


Tables 6 and 7 provide the MLEs of the model parameters, their standard errors (SEs) and the values of $-\hat{\ell}$, KS and PV for both data sets, respectively.

Table 6: Estimated values with SEs (in parentheses), and analytical measures of the KAPW distribution and other competitors for actual taxes data

\begin{tabular}{|c|c|c|c|c|c|c|c|c|}
\hline Distribution & \multicolumn{5}{|c|}{ Estimates (SEs) } & $-\hat{\ell}$ & KS & PV \\
\hline $\begin{array}{l}\text { KAPW } \\
(\alpha, \beta, \lambda, a, b)\end{array}$ & $\begin{array}{c}323.173 \\
(17.699)\end{array}$ & $\begin{array}{c}0.8279 \\
(0.4678)\end{array}$ & $\begin{array}{c}0.9761 \\
(1.0422)\end{array}$ & $\begin{array}{l}16.5278 \\
(31.148)\end{array}$ & $\begin{array}{c}0.2607 \\
(0.2665)\end{array}$ & 187.887 & 0.0617 & 0.9779 \\
\hline $\begin{array}{l}\text { GBXW } \\
(\alpha, \beta, a, b)\end{array}$ & $\begin{array}{l}175.749 \\
(159.08)\end{array}$ & $\begin{array}{l}258.722 \\
(433.25)\end{array}$ & $\begin{array}{c}5.8501 \\
(0.8959)\end{array}$ & $\begin{array}{c}0.0264 \\
(0.0079)\end{array}$ & & 188.349 & 0.0631 & 0.9727 \\
\hline $\begin{array}{l}\mathrm{EW} \\
(\alpha, \beta, \lambda)\end{array}$ & $\begin{array}{l}2813.00 \\
(11683)\end{array}$ & $\begin{array}{c}0.2772 \\
(0.1420)\end{array}$ & $\begin{array}{c}4.2640 \\
(3.5913)\end{array}$ & & & 188.241 & 0.0640 & 0.9686 \\
\hline $\begin{array}{l}\text { OLLEW } \\
(\alpha, \beta, \gamma, \theta)\end{array}$ & $\begin{array}{c}0.0721 \\
(0.1505)\end{array}$ & $\begin{array}{c}0.1500 \\
(0.1433)\end{array}$ & $\begin{array}{c}5.5155 \\
(6.9616)\end{array}$ & $\begin{array}{c}7.0781 \\
(9.9837)\end{array}$ & & 190.718 & 0.0727 & 0.9134 \\
\hline $\begin{array}{l}\text { APW } \\
(\alpha, \beta, \lambda)\end{array}$ & $\begin{array}{r}3432.25 \\
(4219.6) \\
\end{array}$ & $\begin{array}{c}0.8786 \\
(0.0934) \\
\end{array}$ & $\begin{array}{c}0.2811 \\
(0.0792)\end{array}$ & & & 192.019 & 0.1055 & 0.5266 \\
\hline $\begin{array}{l}\text { TCWG } \\
(\alpha, \beta, \lambda, \delta)\end{array}$ & $\begin{array}{c}0.9999 \\
(0.6072) \\
\end{array}$ & $\begin{array}{c}2.0179 \\
(0.3280) \\
\end{array}$ & $\begin{array}{c}0.6436 \\
(0.2337) \\
\end{array}$ & $\begin{array}{c}0.0538 \\
(0.0109) \\
\end{array}$ & & 195.706 & 0.1324 & 0.2518 \\
\hline $\begin{array}{l}\text { ALTW } \\
(\alpha, \beta, \lambda)\end{array}$ & $\begin{array}{c}0.4333 \\
(0.2708)\end{array}$ & $\begin{array}{c}1.9431 \\
(0.1099) \\
\end{array}$ & $\begin{array}{c}0.0039 \\
(0.0014)\end{array}$ & & & 196.466 & 0.1228 & 0.3353 \\
\hline $\begin{array}{l}\text { WW } \\
(\alpha, \beta, a, b)\end{array}$ & $\begin{array}{c}0.2636 \\
(4.9663)\end{array}$ & $\begin{array}{l}77.5484 \\
(23.026)\end{array}$ & $\begin{array}{c}0.6699 \\
(0.1279)\end{array}$ & $\begin{array}{c}0.0170 \\
(0.0070)\end{array}$ & & 197.380 & 0.1432 & 0.1774 \\
\hline $\begin{array}{l}\text { W } \\
(\beta, \lambda)\end{array}$ & $\begin{array}{c}1.8403 \\
(0.1711)\end{array}$ & $\begin{array}{c}0.0653 \\
(0.0049)\end{array}$ & & & & 197.290 & 0.1431 & 0.1780 \\
\hline
\end{tabular}

Table 7: Estimated values with SEs (in parentheses), and analytical measures of the KAPW distribution and other competitors for glass fibers data

\begin{tabular}{|c|c|c|c|c|c|c|c|c|}
\hline Distribution & \multicolumn{5}{|c|}{ Estimates } & $-\hat{\ell}$ & KS & PV \\
\hline $\begin{array}{l}\mathrm{KAPW} \\
(\alpha, \beta, \lambda, a, b)\end{array}$ & $\begin{array}{l}89.2219 \\
(263.60)\end{array}$ & $\begin{array}{c}5.4350 \\
(1.5714)\end{array}$ & $\begin{array}{c}0.1260 \\
(0.0816)\end{array}$ & $\begin{array}{c}0.4975 \\
(0.2354)\end{array}$ & $\begin{array}{c}0.8040 \\
(0.8459)\end{array}$ & 12.150 & 0.0975 & 0.5864 \\
\hline $\begin{array}{l}\text { GBXW } \\
(\alpha, \beta, a, b)\end{array}$ & $\begin{array}{c}0.4623 \\
(0.7270) \\
\end{array}$ & $\begin{array}{c}1.3915 \\
(0.8250) \\
\end{array}$ & $\begin{array}{c}0.0690 \\
(0.2522) \\
\end{array}$ & $\begin{array}{c}2.9125 \\
(2.7039) \\
\end{array}$ & & 14.565 & 0.1406 & 0.1653 \\
\hline $\begin{array}{l}\text { EW } \\
(\alpha, \beta, \lambda)\end{array}$ & $\begin{array}{c}0.6712 \\
(0.2209)\end{array}$ & $\begin{array}{c}7.2844 \\
(1.4869) \\
\end{array}$ & $\begin{array}{c}0.0194 \\
(0.0210) \\
\end{array}$ & & & 14.675 & 0.1462 & 0.1351 \\
\hline $\begin{array}{l}\text { OLLEW } \\
(\alpha, \beta, \gamma, \theta)\end{array}$ & $\begin{array}{c}1.9919 \\
(0.2971)\end{array}$ & $\begin{array}{c}8.7488 \\
(3.9362) \\
\end{array}$ & $\begin{array}{c}0.3021 \\
(0.2664)\end{array}$ & $\begin{array}{c}1.6872 \\
(0.7428) \\
\end{array}$ & & 14.024 & 0.1319 & 0.2223 \\
\hline $\begin{array}{l}\text { APW } \\
(\alpha, \beta, \lambda)\end{array}$ & $\begin{array}{l}10.8558 \\
(12.717)\end{array}$ & $\begin{array}{c}4.4836 \\
(0.7626)\end{array}$ & $\begin{array}{c}0.1947 \\
(0.1082)\end{array}$ & & & 13.474 & 0.1224 & 0.3010 \\
\hline $\begin{array}{l}\text { TCWG } \\
(\alpha, \beta, \lambda, \delta)\end{array}$ & $\begin{array}{c}0.0698 \\
(0.1140) \\
\end{array}$ & $\begin{array}{c}3.2035 \\
(0.9403) \\
\end{array}$ & $\begin{array}{l}-0.1380 \\
(0.9303) \\
\end{array}$ & $\begin{array}{c}0.8911 \\
(0.1952) \\
\end{array}$ & & 12.030 & 0.0995 & 0.5598 \\
\hline $\begin{array}{l}\text { ALTW } \\
(\alpha, \beta, \lambda) \\
\end{array}$ & $\begin{array}{c}22.528 \\
(42.543) \\
\end{array}$ & $\begin{array}{c}4.4786 \\
(0.7487) \\
\end{array}$ & $\begin{array}{c}0.2549 \\
(0.1913) \\
\end{array}$ & & & 13.575 & 0.1432 & 0.1507 \\
\hline $\begin{array}{l}\text { WW } \\
(\alpha, \beta, a, b)\end{array}$ & $\begin{array}{c}0.0278 \\
(0.0724) \\
\end{array}$ & $\begin{array}{c}3.1168 \\
(2.7740) \\
\end{array}$ & $\begin{array}{c}0.8617 \\
(0.5162) \\
\end{array}$ & $\begin{array}{c}1.0134 \\
(0.6708) \\
\end{array}$ & & 14.412 & 0.1373 & 0.1852 \\
\hline $\begin{array}{l}\mathrm{W} \\
(\beta, \lambda)\end{array}$ & $\begin{array}{c}5.7807 \\
(0.5760)\end{array}$ & $\begin{array}{c}0.6142 \\
(0.0139)\end{array}$ & & & & 15.206 & 0.1522 & 0.1078 \\
\hline
\end{tabular}

Plots of the fitted KAPW PDF and other fitted densities, for both data sets, are displayed in Figures 4 and 5 , respectively. The estimated PDF, CDF, SF and PP plots for both data sets are shown in Figure 6. Based on the values in Tables 3 and 4 and the plots in Figures 4, 5 and 6, we conclude that the KAPW distribution provides a close fit to both data sets as compared to other rival distributions. 


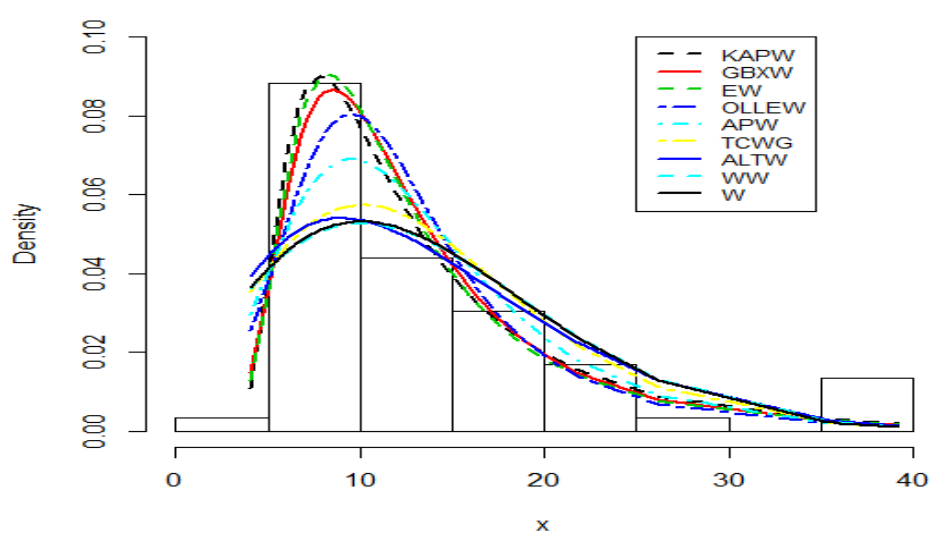

Figure 4: The estimated KAPW PDF and other estimated PDFs for actual taxes data

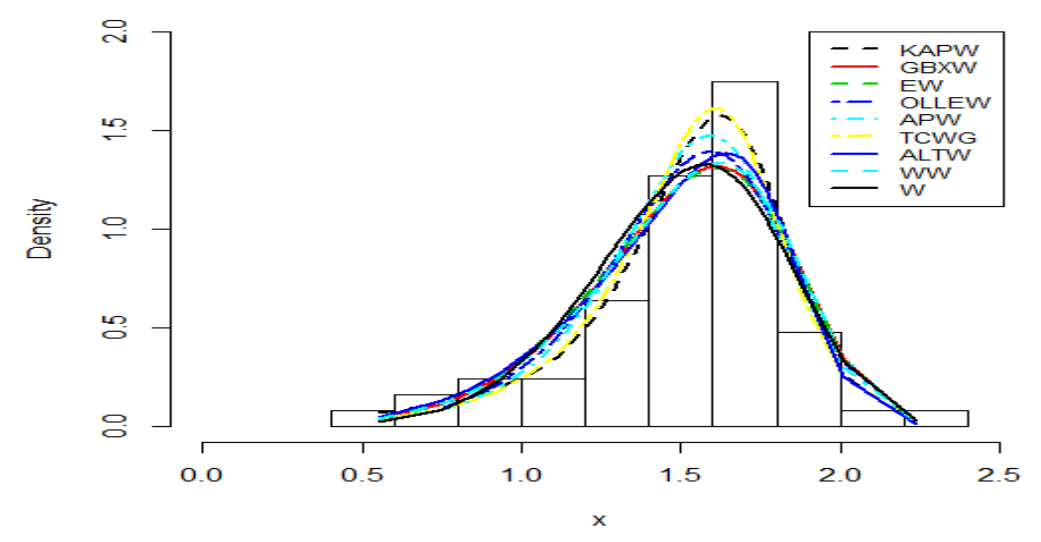

Figure 5: The estimated KAPW PDF and other estimated PDFs for glass fibers data
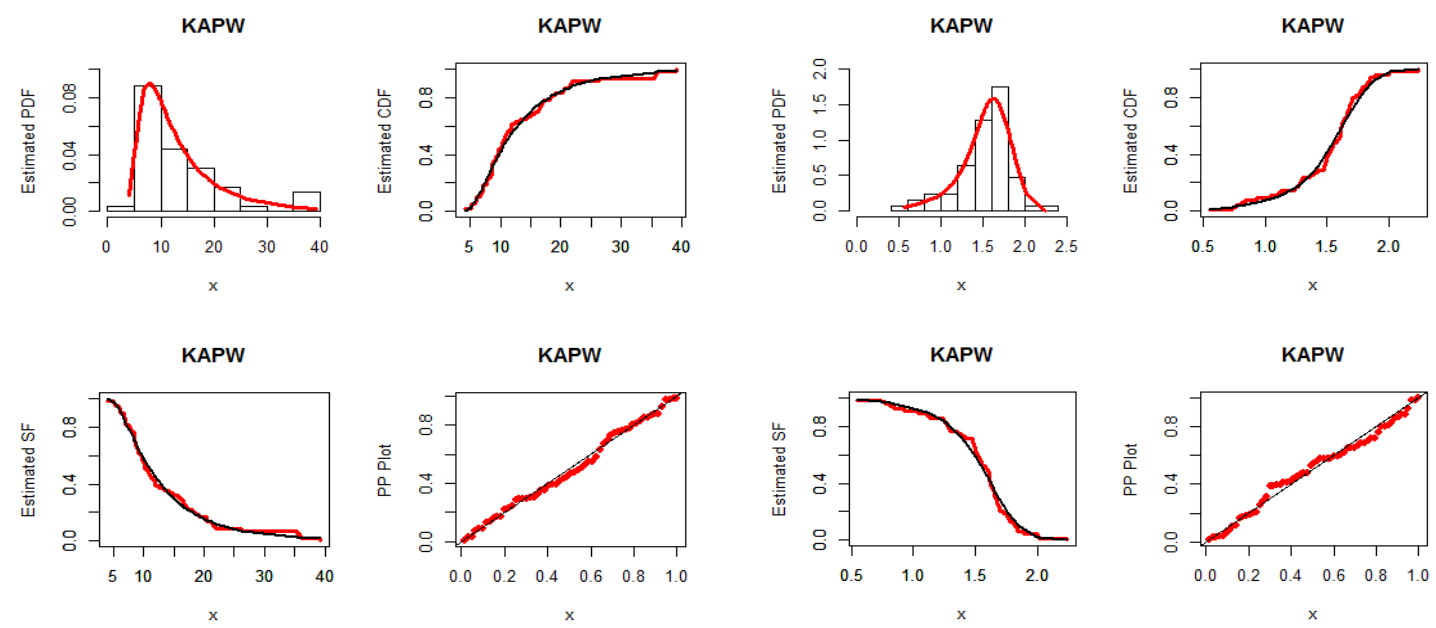

Figure 6: Estimated PDF, CDF, SF and PP plots of the KAPW distribution (left) for actual taxes data and (right) for glass fibers data 


\section{Concluding remarks}

In this article, we introduce the Kumaraswamy alpha power-G (KAP-G) family that extends the alpha power-G (Mahdavi and Kundu, 2017) and Kumaraswamy-G (Cordeiro and de Castro, 2011), exponentiated-G (Gupta et al., 1998) families. Based on the KAP-G class, we construct the four-parameter Kumaraswamy alpha power Weibull (KAPW) distribution which has some desirable properties. Some mathematical quantities of the KAPW model are derived. The KAPW parameters are estimated via maximum likelihood method and detailed simulation results are obtained to assess the performance of the estimates. The flexibility of the KAPW model is examined by two real data applications, proved its better fits as compared to several others Weibull extensions.

Acknowledgments: This work was funded by the University of Jeddah, Saudi Arabia, under grant No. (UJ-02-057DR). The authors, therefore, acknowledge with thanks the University technical and financial support.

\section{References}

1. Abouelmagd, T. H. M., Al-mualim, S., Elgarhy, M., Afify, A. Z. and Ahmad, M. (2017). Properties of the four-parameter Weibull distribution and its applications. Pak. J. Stat., 33, 449-466.

2. Afify, A. Z., Alizadeh, M., Zayed, M., Ramires, T. G. and Louzada, F. (2018). The odd log-logistic exponentiated Weibull distribution: regression modeling, properties, and applications. Iranian Journal of Science and Technology, Transactions A: Science, 42, 2273-2288.

3. Afify, A. Z., Cordeiro, G. M., Butt, N. S., Ortega, E. M. and Suzuki, A. K. (2017). A new lifetime model with variable shapes for the hazard rate. Brazilian Journal of Probability and Statistics, 31, 516-541.

4. Afify, A. Z., Cordeiro, G. M., Yousof, H. M., Alzaatreh, A. and Nofal, Z. M. (2016a). The Kumaraswamy transmuted-G family of distributions: properties and applications. Journal of Data Science, 14, 245-270.

5. Afify, A. Z., Nofal, Z. M. and Butt, N. S. (2014). Transmuted complementary Weibull geometric distribution. Pak. J. Stat. Oper. Res., 10, 435-454.

6. Afify, A. Z., Yousof, H. M., Cordeiro, G. M., Ortega, E. M. and Nofal, Z. M. (2016b). The Weibull Fréchet distribution and its applications. Journal of Applied Statistics, 43, 2608-2626.

7. Aldahlan, M. A. D., Khalil, M. G. and Afify, A. Z. (2018). A new generalized family of distributions for lifetime data. Journal of Modern Applied Statistical Methods. To appear.

8. Alizadeh, M., Afify, A. Z., Eliwa, M. S. and Ali, S. (2020). The odd log-logistic Lindley-G family of distributions: properties, Bayesian and non-Bayesian estimation with applications. Computational Statistics, 35, 281-308.

9. Cordeiro, G. M., Afify, A. Z., Yousof, H. M., Cakmakyapan, S. and Ozel, G. (2018). The Lindley Weibull distribution: properties and applications. Annals of the Brazilian Academy of Sciences, 90, 2579-2598.

10. Cordeiro, G. M. and de Castro, M. (2011). A new family of generalized distributions, Journal of Statistical Computation and Simulation 81, 883-893.

11. Cordeiro, G. M., Ortega, E. M. and Nadarajah, S. (2010). The Kumaraswamy Weibull distribution with application to failure data. Journal of the Franklin Institute, 347, 1399-1429.

12. Cordeiro, G. M., Ortega, E. M. and Lemonte, A. J. (2014). The exponential-Weibull lifetime distribution, Journal of Statistical Computation and Simulation, 84, 2592-2606.

13. Gupta, R. C., Gupta, P. L. and Gupta, R. D. (1998). Modeling failure time data by Lehmann alternatives. Commun. Stat. Theory Methods, 27, 887-904.

14. Jalmar, M. F., Ortega, E. M. and Cordeiro, G. M. (2008). A generalized modified Weibull distribution for lifetime modeling. Computational Statistics and Data Analysis, 53, 450-462.

15. Mahdavi, A. and Kundu, D. (2015). A New Method for Generating Distributions with an Application to Exponential Distribution. Commun. Stat. Theory Methods, 46, 6543-6557.

16. Mead, M. E., Cordeiro, G. M., Afify, A. Z. and Al Mofleh, H. (2019). The alpha power transformation family: properties and applications. Pak. J. Stat. Oper. Res., 15, 525-545.

17. Mudholkar, G. S. and Srivastava, D. K. (1993). Exponentiated Weibull family for analyzing bathtub failurerate data. IEEE transactions on reliability, 42, 299-302.

18. Nadarajah, S., Cordeiro, G. M. and Ortega, E. M. (2013). The exponentiated Weibull distribution: a survey. Statistical Papers, 54, 839-877.

19. Nassar, M., Afify, A. Z., Dey, S. and Kumar, D. (2018). A new extension of Weibull distribution: Properties and different methods of estimation. Journal of Computational and Applied Mathematics, 336, 439-457.

20. Nassar, M., Alzaatreh, A., Mead, M. and Abo-Kasem, O. (2017). Alpha power Weibull distribution: properties and applications. Commun. Stat. Theory Methods, 46, 10236-10252. 
21. Nassar, M., Kumar, D., Dey, S., Cordeiro, G. M. and Afify, A. Z. (2019). The Marshall-Olkin alpha power family of distributions with applications. Journal of Computational and Applied Mathematics, 351, 41-53.

22. Smith, R. L. and Naylor, J. C. (1987). A comparison of maximum likelihood and Bayesian estimators for the three-parameter Weibull distribution. Appl. Statist., 36, 358-369.

23. Xie, M., Lai, C. D. (1995). Reliability analysis using an additive Weibull model with bathtub-shaped failure rate function. Reliability Engineering and System Safety, 52, 87-93.

24. Xie, M., Tang, Y. and Goh, T. N. (2002). A modified Weibull extension with bathtub failure rate function. Reliability Engineering and System Safety, 76, 279-285. 